\title{
An Empirical Study on Teaching Strategies of Chinese Classifiers under Multicultural Background
}

\author{
Yanan Gao ${ }^{1 \mathrm{a}}$ and Hongfei Liu $\mathrm{bb}^{\text {* }}$ \\ ${ }^{1}$ Shenyang Aerospace University No.37Ave Daoyi, Shenyang, China \\ ${ }^{2}$ Liaoning University No. 66 Ave Chongshan, Shenyang, China \\ 1'gaoyanan05111031@163.com \\ 2 185741670@qq.com
}

Keywords: Classifiers' teaching; Multiculturalism; Universality introduction; Empirical research

\begin{abstract}
Based on the universalities of the world languages and the general cognitive characteristics of human beings, the paper explores the effective teaching strategies of Chinese classifiers. It proposes that put measure words as the teaching point of Chinese classifiers, takes the shape feature as the breakthrough and important parameter of classifiers' matching motivation, and adopts "source - prototype association " teaching strategy to the complex semantic classifiers. Experiments show that those strategies are effective teaching methods for foreign students to learn Chinese classifiers.
\end{abstract}

\section{Introduction}

At present, nearly 400 thousand foreign students from more than 200 countries have been studying in China. They come from different cultures around the world. How to teach effectively in a multicultural background is a big problem faced by the Chinese international education. However, the mandatory and weak rule of Chinese classifiers make them difficult and important in international Chinese teaching. In view of those, this study tries to use the ontology research results, applies the latest theoretical researches to the international teaching of Chinese, in order to find the generalities and differences of Chinese classifiers in the world classification systems, based on the commonness of the world languages and the universal cognitive characteristics of human beings. It explores the impactful teaching strategies of Chinese classifiers to improve the teaching effectiveness and promotes Chinese language and culture to go out better.

\section{The Strategy of Measure Words First}

Through the study of the 500 languages in the world, Aikhenvald (2000) denotes that the classifiers are not only the characteristic of the Chinese and Tibetan language, the distribution of classifiers widely in the languages of South Asia, Indo European and even Africa countries. In particular, Chinese classifiers belong to one of the number-classifiers system. The decomposition analysis of the classifiers characteristics of He Xiaowei and Cai Jilang (2015) find that the noun relying on classifier for implementing the semantic operation of metrological atom is a common feature of the world languages, while the different characteristics of the Chinese classifiers and the English plural affixes are the divergences from the number and classification features. That is, the characteristics of CL can be divided into two kinds, the first kind is [Indiv(iduate)], which is an inherent and mandatory feature of CL. It has the function of individualization of nouns. The second kind is composed of $[ \pm \mathrm{Num}]$ and $[ \pm \mathrm{Cla}$ (ssification)], which are not mandatory. The Chinese CL chooses [+ Cla] features, and English CL chooses [+ Num] features generally. Li Xuping (2013) 
also points out that there is no distinction between classifiers and measure words in Chinese, that is to say measurement scales, container and individual classifiers are both determination of a counting unit intrinsically. Their difference lie in the former including a certain amount itself and the latter containing classified characteristics.

In view of this, in the international teaching of Chinese, we propose to take measure words as a breakthrough point in the teaching of classifiers, and suggest translating classifiers into "unit of" rather than "measure word". In other words, the measure words in the world languages, such as the unit "Yuan" and the weight unit "Jin", are used as the starting point for the teaching of Chinese classifiers, and then introduce gradually individual classifiers and verbal classifiers. "Unit of" is more appropriate for classifier in Chinese international textbooks and more suitable for foreign students to understand. "Measure word" is only literal translation of classifier, which is only acceptable for expressing partial classifiers, and dose not reveal the essential characteristics of Chinese classifiers.

To investigate the effectiveness of this teaching strategy, two primary Chinese parallel classes (a total of 80 people) were selected for comparative research. We use the method of qualitative and quantitative analysis, such as test, questionnaire, interview and classroom observation, to analyze the data collected from the three dimensions of the correct rate of written examination, the error rate of oral examination questions and the speed of answer. The results are as shown in 1 and 2, whether they are the average rate of correct questions and the speed of answering questions, the 1 classes using this strategy were higher than the 2 classes of regular teaching, especially in the efficiency of acquisition. The error rate of the 2 class was significantly higher than that of class 1 .

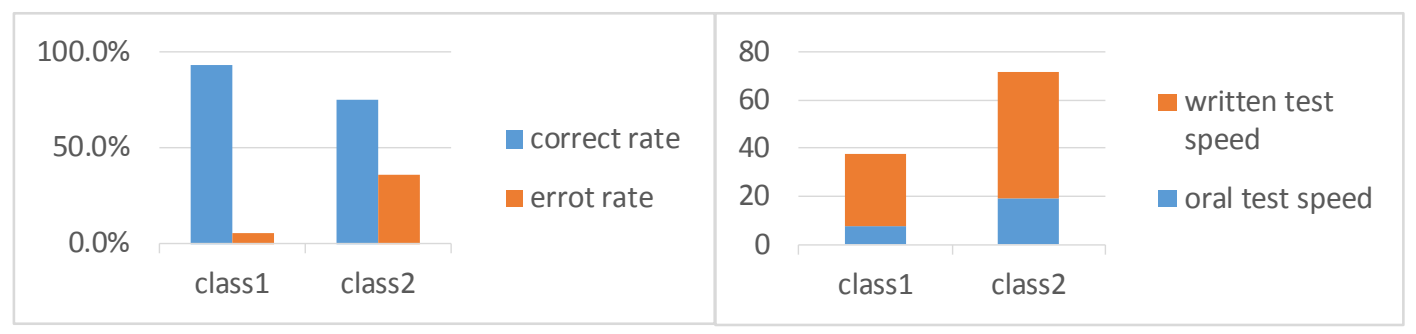

Figure 1.

Figure 2.

\section{Shape Features are Important Parameters for Classifiers' Matching}

Allan (1977) and Aikhenvald (2000) induced the semantic parameters used by classifiers in the world language. "Animacy" and "shape" features are the two basic semantic parameters of the classifiers, and for inanimate objects, shape is the first semantic parameter. Kuo (2009) experimental study shows that Chinese classifiers pay more attention to establishing semantic choice relationship from the appearance

features of things, and the influence of shape on Chinese people is far higher than that of British people. Erbaugh (1986) children's acquisition research shows that shape feature classifiers acquisition are earlier than material, function and other characteristic classifiers, and shape is the most important parameter for the extension of classifiers. Adams, Conklin(1973) also explained it, the classification of classifiers is closely related to human cognition, and vision is more susceptible to sense of smell, hearing, taste and sensation. Therefore, the features of things are the most important basis for classifiers classification.

The individual classifiers are the most common classifiers in Chinese as well as the focus of the 
foreign students' acquisition of classifiers. The key to the teaching them are to reveal the motivation of classifier matching, so that the students not only to know them but also to get the reason when they are learning classifiers. Therefore, the Chinese international teachers can use the picture or the physical method for inspiring students to find the shape characteristics of the classifiers. Take classifier "Zhang" as an example, through the display of the pictures of "bow " and "mouth", students can find out the appearance features of the two things that can be extended when they are used, and then by displaying "newspaper" "bank card" "bed" and other things to help students' memory the second uses of classifier "Zhang", which matches the plane things, and finally give the "one Zhang "construction to let student associate other things with the same shape characteristics.

In order to investigate the influence of the shape characteristics on the acquisition of classifier, the questionnaire of the same class (60 people) was adopted. The questionnaire was divided into two sets of A and B. The difference isway of asking questions. One is to give the specific context by the sentence which help students choose the appropriate classifier. The other one is to use classifier's shape feature by picture form to help students choose it. The two sets of papers are used alternately, that is, the first overseas student gets A volume, and the second student gets B volumes, and so forth. As shown in 3, the correct area of the shape of the table is obviously higher than that of the table context, especially the classifier "tiao" ,"pian" and "pai". From the given picture, we can clearly see the shape features of long thing, thin and flat thing and standing on a row. The ratio between shape and context are up to 2:1, 5:3 And 7:4. That is to say, the shape characteristics of the matching things will have a great influence on the acquisition of dynamic classifier. The Chinese teachers can make full use of the shape characteristics.

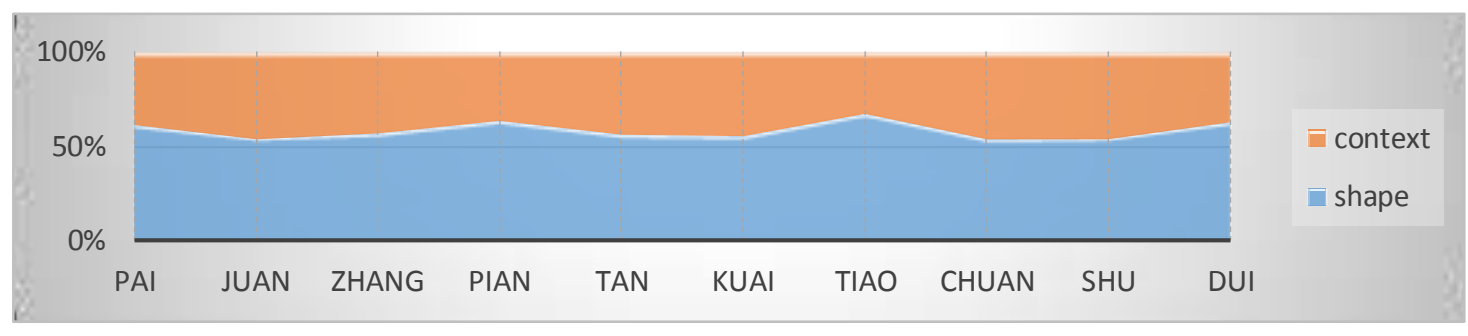

Figure 3.

\section{Source-Prototype Association Strategy}

The development of language has caused the generalization of means of classifiers, which result in more difficult to understand between classifier and object. However, cognitive research shows that classifier is an important category of nouns, and all objects are composed a prototype category marked by the classifier (Lakoff1987, Zong Shouyun 2008). The study of grammaticalization shows that like other languages in the world, the Chinese classifier is derived from the noun and the verb, to a certain extent, it still maintains the semantic features of the source meaning. At the same time, Bisang (1999) also points out that Chinese classifier is an item- oriented, the meaning of classifier is derived from the specific semantics of a single individual object, and is expanded further according to this semantic feature. For example, the classifier "bo" derives from the verb "allocate" meaning, which still exists in modern Chinese, such as allocate funds and grain allocation, thus "bo" in the classifier structure of "dividing into two dials" also highlights the action process of dividing the crowd apart. 
The complex semantic classifiers are difficult point in the teaching of classifiers. This kind of words need to be built semantic association one by one instead of the whole systematic explanation. Based on the above results, we propose "source-prototype association" teaching method, which build the classifier matching motivation through the original meaning, and establish the semantic relation between the classifiers and the atypical members with the help of the prototype members. The study of Rosch, E. also shows that typical members have the function of conceptual representation. We always understand concepts through the best examples of concepts. The related experiments of cognitive psychologists also show that people have the psychological tendency to use prototypes in the process of conceptual mastery. If people understand the information of the prototype, they have more reasonable judgment naturally on their variants, and they are not easy to occur cognitive deviations. The American educational cognitive psychology Ausubel, D.P. also put forward a famous meaningful learning theory. This theory holds that school learning is mainly meaningful acceptance learning. It is a process of conceptual assimilation under the guidance of teachers. That is to say, teachers should try to guide students to build the relationship between new knowledge and old knowledge in the cognitive structure of the learners so that the knowledge can be successfully enter the knowledge structure of the learners.

In order to verify the teaching effect of this teaching strategy, we let the students to choose the correct choice of the "fu" and "chuan" in the form of multiple topics, and then teach the classifier in semantic relevance according to the teaching steps above, finally let the students do the two questions again and compare the two results. As in 4 and 5:

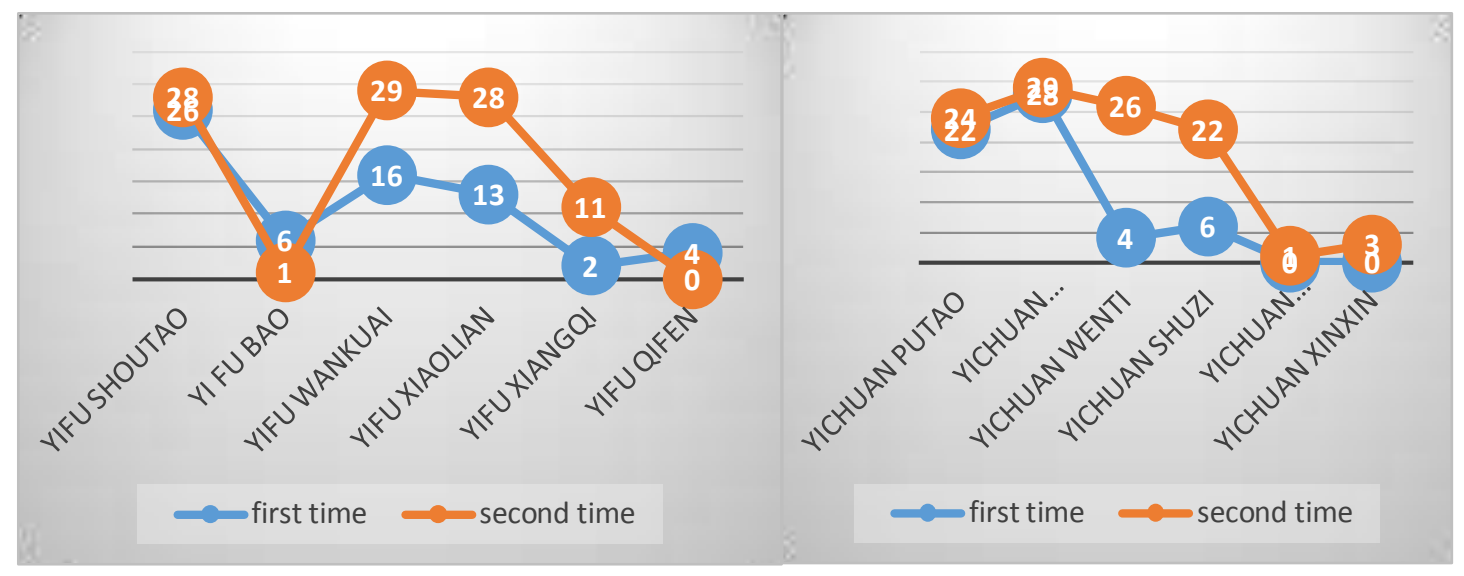

Figure 4.

Figure 5 .

The A, C, D and E options in 4 are the correct answer of the classifier "fu", and the B and F options are wrong. The A, B, C and D in 5 are the correct answer of the classifier "fu", and E and F are the wrong. As we can see from figures, the accuracy of the second time is obviously better than the first, that is, the second right node is on the first, and the second fault node is located under the first. Similarly, in the acquisition chart of the classifier "chuan", the second fold line is higher than the first, especially in the atypical usage of the node $\mathrm{C}$ and $\mathrm{D}$. At the first time, the number of foreign students who choose "a series of problems" and "a string of figures" is 4 and 6 respectively, while the number of second time increases to 26 and 22. It can be seen that the strategy of semantic association teaching has an important influence on the teaching of the atypical usage of complex semantic classifiers. In a word, from the results of the survey, it is an effective teaching strategy for the complicated semantics classifiers in teaching Chinese as the second language. 


\section{Reference}

[1] A. Y. Aikhenvald, Classifiers: A Typology of Noun Categorization Device.Oxford: Oxford University Press, 2000.

[2] W. Bisang, Classifiers in East and Southeast Asian language Counting and beyond. L.S. Cheng, \& R. Sybesma, Bare and Not-So-Bare Nouns and the Structure of NP, Linguistic Inquiry, 1999.

[3] J. Z. Paul, \& Z. Grüter, Blocking effects in the learning of Chinese classifiers, Language Learning, 1(2016).

[4] Y. H.Li, Argument determiner phrases and number phrases, Linguistic Inquiry 29(1998).

[5] X. P. Li, Numeral Classifiers in Chinese, Berlin: Mouton de Gruyter,2013.

[6] P. Li, \& B. Huang, \& Y. Hsiao, Learning that Classifiers Count: Mandarin-Speaking Children's Acquisition of Sortal and Mensural Classifiers. Journal of East Asian Linguistics, 19(2017).

[7] J.Z. Paul, \& Z. Grüter, Blocking effects in the learning of Chinese classifiers, Language Learning, 1 (2016).

[8] H.Y. Tai, Variation in Classifier Systems Across Chinese Dialects: Towards a Cognition-Based Semantic Approach, Chinese Language and Linguistics 1: Chinese Dialects, 1992.

[9] W.T. Tsai, On Subject Specificity and Theory of Syntax-Semantics Interface. Journal of East Asian Linguistice, 10(2015).

[10] Zhang, N. N. Classifiers Structures in Mandarin Chinese. Berlin: Mouton de Gruyter, 2013. 